\title{
A Multivariate Examination of Active and Interactive Learning and Student Engagement in Post-Secondary Engineering Energy Science Classrooms: The "Why" of Instructional Strategy Use
}

\section{Dr. Jonathan C. Hilpert, Georgia Southern University}

Dr. Jonathan C. Hilpert is an Associate Professor of Educational Psychology in the College of Education (COE) at Georgia Southern University. His research focuses on examining student engagement and interactive learning in post-secondary STEM courses from a complex systems perspective, including the emergent and dynamic qualities of psychological and collaborative systems and their underlying network structures. His research findings have been disseminated in national and international engineering education and psychological journals (including Journal of Engineering Education, Journal of Women and Minorities in Science and Engineering, Educational Psychology, Psychological Inquiry, and Motivation and Emotion) as well as presented at national and internal conferences (including American Education Research Association, American Society for Engineering Education; European Association for Research in Learning and Instruction; and various symposiums organized by the National Science Foundation). Dr. Hilpert teaches graduate and undergraduate courses in the COE, including topics such as learning theories and applications, thinking and problem solving, interactive learning, models of motivation, assessment and classroom management, and field based research methods. He has expertise in a range of research methods and analytical techniques, including structural equation modeling, hierarchical linear modeling, longitudinal growth curve modeling, measurement and assessment of latent constructs, thematic analysis, and network analysis. 


\title{
A Multivariate Examination of Active and Interactive Learning and Student Engagement in Post-Secondary Engineering Energy Science Classrooms: The 'Why' of Instructional Strategy Use
}

\begin{abstract}
Recent national STEM initiatives have shifted research focus from the development of instructional innovations to the examination of change processes and implementation of research based instructional strategies. Emphasis has been placed on adoption of instructional strategies and how they are implemented, especially in engineering science courses at the core of engineering curriculum. Unfortunately, little has been done to examine the multivariate relationship among instructional strategies, active and interactive learning, and student engagement in post-secondary engineering energy science courses. Successful implementation of instructional strategies hinges not only on the how, but also the why and for what purpose. The current study provides evidence for the complex, multivariate relationship among eight instructional strategies, active and interactive learning, and multiple forms of student engagement in post-secondary engineering energy science courses. Results have implications for the specific use of instructional strategies to promote different forms of engagement and learning. This material is based upon work supported by the National Science Foundation under Grant No. 1245018.
\end{abstract}

\section{Introduction}

The study of instructional strategies and active learning in higher education STEM classrooms has deep roots (Freeman, et al., 2014; Henderson, Beach \& Finkelstein, 2011; McKeachie \& Svinicki, 2014; Prince, 2004) but little has been done to examine the multivariate relationship between instructional strategies, active and interactive learning, and student engagement in postsecondary engineering education. From a practical standpoint, it is important to understand the relationship between instruction, active learning, and engagement so that strategies can be tailored to provide appropriate learning experiences for students. Different forms of instructional strategies (Borrego, et al., 2013) may engage students in different ways, producing different cognitive, affective, and behavioral processes better suited for one type of learning over another.

Engineering education researchers have examined types of instructional strategies adopted and implemented by engineering programs across the country (Borrego, Froyd, \& Hall, 2010; Borrego, et al., 2013). Too, engagement researchers have operationalized cognitive, affective, and behavioral forms of engagement (Corno \& Mandinach, 2004; Fredricks, Blumenfeld \& Paris, 2004) as well as examined interactive forms of engagement (Hake, 1998; Hilpert \& Husman, 2015). These forms of engagement represent different overlapping dimensions of a larger nomological network of engagement constructs, or a network of proposed thoughts, behaviors, and feelings that comprise how students carry out their school work (Lawson \& Lawson, 2013). The purpose of this paper is to examine the multivariate relationship among different forms of instructional strategies and different forms of learning and engagement in post-secondary engineering energy science classrooms.

Recent national STEM initiatives have shifted their focus from the development of instructional strategy innovations to the examination of change processes and implementation of instructional strategies (NRC, 2012). Emphasis has been placed on adoption of instructional strategies and 
how they are implemented, especially in engineering science courses at the core of engineering curriculum (Borrego, et al., 2013). This study examines eight instructional strategies and how their implementation produces different active and interactive learning experiences and engagement patterns. The results provide empirical evidence for the pattern and strength of relationship between different instructional strategies, active and interactive learning, and different forms of engagement in engineering education classrooms. The results have implications for why and what purpose instructional strategies can and should be used in engineering science courses based on what impact they have on student learning and engagement.

\section{Active and Interactive Learning}

Over the last two decades, there has been a well-documented push to increase so-called active learning in post-secondary STEM classrooms (Felder \& Brent, 2009; Prince, 2004). Faculty are regularly encouraged to incorporate instructional strategies into their teaching repertoires that, presumably, improve their students' learning experiences by making them more active (Henderson, Beach \& Finkelstein, 2011; McKeachie \& Svinicki, 2014). However, what is meant by active learning is not terribly consistent from one study or recommendation to the next and many different types of education experiences fall under the umbrella of active learning (Hilpert \& Husman, in press). In response to this ambiguity, Chi (2009) examined the terminology and various meanings behind the differences between active and interactive learning, providing a clearer picture of the concept. Below the differences between active and interactive learning are briefly reviewed. Then, these concepts are applied to instructional strategy use in engineering education.

Active Learning in Post-Secondary STEM Classrooms. In the post-secondary STEM education literature researchers have tended to conflate active (Felder \& Brent, 2009) and interactive (Hake, 1998; Hilpert \& Husman, 2015) learning under the umbrella term active learning, which encompasses many types of instructional strategies (Hilpert \& Husman, in press). Instructional strategies ranging from the use of clickers (Caldwell, 2007) to cooperative quizzes (Zeilik \& Morris, 2004) to classroom group work (Knight \& Wood, 2005) with both active and interactive attributes have been combined to carry out examinations of active learning (Freeman, et al., 2014; Gasiewski, et al., 2012; Prince, 2004). However, according to Chi (2009) active and interactive learning have different cognitive and behavioral characteristics. Active learning is attending to or selecting relevant information (such as taking notes during lecture or using "clickers") and interactive learning is collaborating to solve problems and receive feedback (such as working in a group to solve a problem). Using classroom observation protocols, Hilpert \& Husman (in press) found that within STEM classrooms, effective instructors tend to use active learning to activate prior knowledge at the beginning of class and interactive learning to encourage application and other higher order thinking skills during class.

Innovative Instruction in Engineering Education. The push for improved post-secondary STEM pedagogy is mirrored, if not heightened, within the context of engineering education (ASEE, 2009; NSF, 2008). Recently, the drive to develop and research new instructional innovations has given way to the call to more fully examine how instructional strategies can and should be more widely implemented from context to context. Borrego and colleagues (2010) reviewed various efforts to promote widespread instructional change within post-secondary engineering education using innovation diffusion theory. Using Rodgers (2003) stage-like framework for studying how 
innovations are adopted within economies (i.e. from initial awareness to adoption), these researchers examined the adoption of innovative instructional strategies in engineering education. In their survey of engineering education department chairs, Borrego and colleagues found that engineering department chairs reported the adoption of classroom group work type strategies (e.g. working in pairs to answer questions) was highest, hovering around $70 \%$ across engineering disciplines. It is still unclear whether these reported adoption rates match classroom practices of engineering educators, and whether these instructional strategies map onto accepted psychological definitions of active and interactive learning (Chi, 2009).

However, their work created an initial framework of innovative strategies that was later incorporated into further examination of other research based instructional strategies (Borrego, et al., 2013). Many of Borrego and colleagues (2010) categories fall under the broad use of the term active learning as it emerged in the STEM education literature, but could more aptly be categorized as interactive (Chi, 2009). Other aspects of the framework such as the use of summer bridge programs, fall outside of the purview of instructional strategies. Furthermore, many strategies related to peer interaction were combined into a single active learning category, and traditional strategies such as the use of lecture or guided practice, not often touted by reformists, are not included. For the current study, Borrego and colleagues (2010) innovative instruction categories were modified to examine student perceptions of faculty instructional strategies. To adapt Borrego and colleagues (2010) framework, categories that were not directly related to instructional strategies (for example, implementing summer bridge programs) were removed. Category names and descriptions were also modified to align with Chi's (2009) active/ interactive framework (for example, the "active learning" category was changed to "classroom group work" and lecture and guided practice were added to our list). The final list included eight instructional strategies (see Table 1).

Table 1

Categories of instructional approaches

\begin{tabular}{|c|c|}
\hline Instructional Strategies & Descriptions Used to Build Survey \\
\hline 1 Classroom Group Work & $\begin{array}{l}\text { Working in pairs or groups to address questions about the material, and } \\
\text { working in pairs or groups to answer problems or challenges that have been } \\
\text { posed by the instructor. }\end{array}$ \\
\hline 2 Artifact Dissection & $\begin{array}{l}\text { Students work together to disassemble a common product (e.g., sewing } \\
\text { machine. bicycle) to explore function and design. }\end{array}$ \\
\hline 3 Service Projects & $\begin{array}{l}\text { Service-learning projects are working with not-for-profit organizations in } \\
\text { the local community to solve problems or help young people. }\end{array}$ \\
\hline 4 Design Projects & $\begin{array}{l}\text { These projects feature individual or team design projects as a significant } \\
\text { part of the class. Students are required to design new approaches to } \\
\text { problems. }\end{array}$ \\
\hline 5 Build Projects & $\begin{array}{l}\text { These projects feature individual or team build projects as a significant part } \\
\text { of the class. Students are required to build something new (e.g. bridge, } \\
\text { robot, website, circuit, etc.). }\end{array}$ \\
\hline 6 Interdisciplinary Design & $\begin{array}{l}\text { Students from multiple disciplines (e.g. engineering, business, } \\
\text { social sciences, sciences, humanities) work together on design projects. }\end{array}$ \\
\hline 7 Lecture & $\begin{array}{l}\text { Students listen to instructor as he or she presents power point slides, writes } \\
\text { on a chalk/white board, or presents notes. }\end{array}$ \\
\hline 8 Guided Practice & $\begin{array}{l}\text { Students work along with professor as he or she demonstrates how to } \\
\text { perform calculations, write programming code, or follow procedures in a } \\
\text { step-by-step fashion. }\end{array}$ \\
\hline
\end{tabular}

Note. Instructional approaches adopted from Borrego, Froyd, \& Simini (2010). Categories not relevant to active or interactive pedagogies removed from original framework. Lecture and guided practice categories added. 
These strategy descriptions were used to create survey items for student self-report measures (example items are including in the measures section below). The first six instructional approaches align with Chi's (2009) descriptions of interactive learning, and the last two align with Chi's (2009) descriptions of active learning. Although not entirely comprehensive according to more recent accounts (Borrego, et al., 2013), the categories likely reflect many forms of instructional strategies students engage with in their engineering courses and can be used to conduct a multidimensional examination of classroom instruction.

\section{Student Engagement}

The study of student engagement exists at multiple level of analysis, and includes examination of institutional, interactive, and individual level variables. For example, Kuh and colleagues (e.g. Kuh, 2002) have systematically studied institutional level student engagement, focusing on factors related to general experiences at university (e.g. involvement in extracurricular activities). Other approaches to the study of student engagement have focused on classroom and individual level factors. For example, educational psychologists have defined individual engagement along behavioral, cognitive, and affective dimensions (Corno \& Mandinach, 2004; Fredricks, Blumenfeld \& Paris, 2004). Other researchers have focused on interactive forms of engagement (e.g. Hake, 1998), or properties of student interaction that emerge during engagement in group problem solving rather than purely individual experiences (Hilpert \& Holliday, 2015; Hilpert \& Husman, 2015). Below we briefly review individual and interactive operationalizations of student engagement.

Individual Student Engagement. At the student level of analysis, educational psychologists have typically operationalized student engagement as a multidimensional psychological construct, with overlapping behavioral, cognitive, and affective dimensions (Wang, Bergin, \& Bergin, 2014). Behavioral engagement refers to observable behaviors related to classroom participation (e.g. asking questions, time on task). Cognitive engagement refers to cognitive variables and processes related to conscious knowledge construction (e.g. concentration, learning strategies). Affective engagement refers to the emotions students experience during classroom learning (e.g. enjoyment, enthusiasm). Wang, Bergin, and Bergin (2014) developed a multidimensional scale to measure these three forms of student classroom engagement, producing validity and reliability evidence for their items, including a distinct disengagement dimension, that measures students tendency to be "zoned out" or thinking about other things rather than school work.

Interactive Student Engagement. Another way of operationalizing student engagement is to approach it as an interactive variable (i.e. Hake, 1998). Instead of examining engagement from a psychological perspective, engagement can be studied from a systems perspective (i.e. Pentland, 2014) at a group level of analysis, with overlapping complex and adaptive interactive engagement dimensions (Hilpert \& Husman, 2015). Complex engagement refers to connected groups of students working together in multiple ways to solve problems. It involves effective communication among students and capitalizing on each other's strengths. Adaptive engagement refers to student groups adjusting to problems spaces and exploring multiple competing ideas. It involves the evolution of possible solutions to problems within student groups, where ideas are explored and vetted for adequacy. Interactive engagement properties are emergent; they are characteristics of collaboration that cannot be reduced to any single student.

\section{The Relationship between Instruction and Engagement}


Taken together, the research that examines the impact of instruction on student engagement in engineering education suggests that although quality of instruction does matter (Chen, et al., 2008; Ohland, et al., 2008; Seymour \& Hewitt, 1997) the positive statistical relationship is small (Hilpert \& Husman, in press). Engagement research assumes a causal mechanism, where instructional strategies facilitate student engagement, producing improved outcomes. Those studies that have examined the relationship between instruction and engagement in postsecondary STEM classrooms have produced small effect sizes. For example, using student selfreport measures, Marchand and Gutierrez (in press) found weak or no relationship between active instruction and engagement in an analysis of graduate statistics courses. Using a combination of self-report and observation protocols, Hilpert and Husman (in press) report effect sizes of $3 \%$ to $7 \%$ for active and interactive instruction on cognitive and behavioral engagement, respectively, in a sample of undergraduate engineering courses. Gasiewski and colleagues (2012), in a similar sample of engineering students, report a $4 \%$ effect size for the impact of active instruction on behavioral engagement.

Work remains to understand the impact of instruction on student engagement. Wang, Bergin, and Bergin (2014), echoing recommendations from other engagement researchers (Fredricks, et al, 2004; Skinner, et al., 2008) suggest that multidimensional examinations of instruction and engagement are needed to fully understand how different forms of instruction and engagement combine to influence student learning. Wang, Bergin, and Bergin (2014) argue that understanding of how engagement functions in the classrooms may be limited because researchers tend to focus on one engagement construct at time. Hilpert and Husman (in press) offer that effect sizes may be artificially small because of measurement reasons; focusing on single individual level constructs may not provide a full enough picture of dynamic classroom processes. Moreover, observation protocols that are calibrated to external observers may not be reflective of student's perceived experiences, introducing large amounts of error into the measurement process. Taken together, research suggests that multivariate studies of engagement and instruction may help to produce better evidence for the complex relationship between different forms of active/ interactive learning and the multi-level, multidimensional engagement construct.

\section{Research Questions}

1) Do students' self-reported mean levels of instruction strategies align with previous findings regarding the adoption rates of engineering educators?

2) Do common forms of instructional strategies in engineering education map onto definitions of active and interactive learning?

3) What is the multivariate relationship between active and interactive learning and individual and interactive engagement?

a. What is the engagement profile related to active learning? What are the strengths and directions of the relationships between active learning and different forms of engagement?

b. What is the engagement profile related to interactive learning? What are the strengths and directions of the relationships between inactive learning and different forms of engagement?

\section{Method}

\section{Procedures and Participants}


Participants were undergraduate engineering students from two participating research universities. Student were enrolled in engineering science courses focused on energy. Example courses include fluid mechanics, thermodynamics, heat transfer, and renewable energy. A stratified sampling technique was utilized, where specific engineering courses were targeted and matched between participating institutions, and then students were recruited from those classrooms. Researchers visited courses and invited to take an online survey to earn a $\$ 10.00$ incentive that was transferred to their student ID cards via a negotiated agreement with university card services. After being recruited, students had one week to log in and complete the online survey. Upon entry into the survey, students provided informed consent. After this, they provided written responses to four open ended questions about their common classroom experiences to prime them for the survey items. After the open ended questions, they responded to Likert scale items to assess their perceptions about instructional strategies and qualities of engagement. The survey items are described in the measures section below.

A total of 346 students participated in the data collection, recruited from a total of 13 engineering energy science courses. Students represented a range of engineering majors, including aerospace, civil, mechanical, industrial, biomedical, electrical, and energy science pathways. Courses were numbered according to typical American conventions, and were at the 200 (68.3\%), 300 $(20.8 \%)$, and $400(10.9 \%)$ levels. Forty four percent of the data came from students enrolled at a university in the southeast, and $56 \%$ of the data came from students enrolled at a university in the southwest. A previous analysis revealed that student responses to the items were invariant across institution (Hilpert \& Husman, 2016). Students were in their sophomore, junior, and senior years in school. The demographic breakdown of the sample was as follows: $52 \%$ White, $11.3 \%$ Hispanic; $10.4 \%$ Black, $6.9 \%$ Asian, $2.6 \%$ two or more races, 1.4\% nonresident, .9\% American Indian, .3\% Pacific Islander, and 14.2\% unknown or not reported. Fifteen percent of the respondents were female.

\section{Measures}

Active and Interactive Learning. Student perceptions of instructional strategies were measured using items adapted from Borrego and colleagues (2010) framework of innovative instruction in engineering courses. Items reflected eight instructional strategies, classroom group work, artifact dissection, service projects, design projects, build projects, interdisciplinary design, lecture, and guided practice, one item for each type. Instructional strategies are aligned with Chi's (2009) definitions of active and interactive learning. Example interactive learning items are, "working on in-class activities with other students (e.g. to answer questions)" and "working in teams to build new things (e.g. bridge, robot, etc.)." An example active learning item is, "working along with the professor as he or she demonstrates how to do something in front of the class (e.g. solve a problem)." Students responded on a seven point Likert scale ranging from never to always.

Individual Engagement. Student perceptions of their cognitive, behavioral, and affective engagement, as well as their disengagement, were measured using Wang, Bergin, and Bergin's (2014) Classroom Engagement Inventory (CEI). An example cognitive engagement item is (8 items; $\alpha=.83$ ), "I ask myself questions as I go along to make sure the work make sense to me." An example behavioral engagement items is ( 5 items; $\alpha=.80)$, "I do not want to stop working at the end of class." An example affective engagement item is ( 5 items; $\alpha=.91$ ), "I feel interested." An example disengagement item is ( 3 items; $\alpha=.78$ ), "I am 'zoned out,' not really 
thinking or doing class work." Students responded on a seven point Likert scale ranging from never to always.

Interactive Engagement. Student perceptions of complex and adaptive interactive engagement were measured using Hilpert, Husman, and Stump's (2015) Innovative Engagement Scale (IES). Example complex engagement items ( 8 items; $\alpha=.95)$ are, "Students in my group had different strengths" and "Over time we established a system for working together." Example adaptive engagement items ( 8 items; $\alpha=.94$ ) are, "Ideas evolved as my group worked together," and "As we worked together, we considered lots of possibilities." Students responded on a seven point Likert scale ranging from not accurate to very accurate, a degree of fidelity to how the items matched their classroom experiences working with other students during classroom activities.

\section{Analysis}

Research Question 1. Data analysis began with calculating descriptive statistics and performing statistical assumptions checking. Mean levels of the instructional strategies were analyzed and qualitative comparison of the student self-reported rates to those published in the extant literature (i.e. Borrego et al., 2010) was conducted.

Research Question 2. An exploratory factor analysis of the instructional strategy items was conducted to produce evidence for the internal reliability and structural validity of students responses to the items and to determine if they map onto the hypothesized active and interactive latent dimensions. The data were examined using IBM SPSS v21. The dimensionality of the items was analyzed using maximum likelihood factor analysis (see Green \& Salkind, 2011 for procedures). We used principle axis extraction with a varimax rotation as this is appropriate for Likert scale data with oblique factors (Tabachnik \& Fidell, 2007). Three criteria were used to determine the number of factors to rotate: our a priori hypothesis about the number of underlying factors, the scree test, and the interpretability of the factor solution. Item scores for individual instructional approaches were parceled into two latent constructs: active and interactive learning.

Research Question 3. Bivariate correlation coefficients were calculated among all study variables to produce evidence for external validity within a nomological network of engagement, active, and interactive learning constructs and to produce initial univariate evidence for all possible correlational relationships among the study variables. The data were examined using IBM SPSS v21. Structural Equation Modeling (SEM, Byrne, 2006) was conducted to provide evidence for the multivariate relationships among the study variables. A structural path analytic model (Byrne, 2006), including the two active and interactive learning measured variables, the four individual engagement measured variables, and the two interactive engagement measured variables was specified (see Figure 1). Data were analyzed using EQS 6.2. The active and interactive learning constructs were positioned as the dependent variables, and the engagement constructs were positioned as the independent variables, such that instruction was predicted from engagement. This allowed for the covariance among the engagement measured variables to be included as a structural component of the model. The parameter estimates predicting active and interactive learning from the engagement variables were analyzed for direction and strength to produce evidence for engagement profiles. 


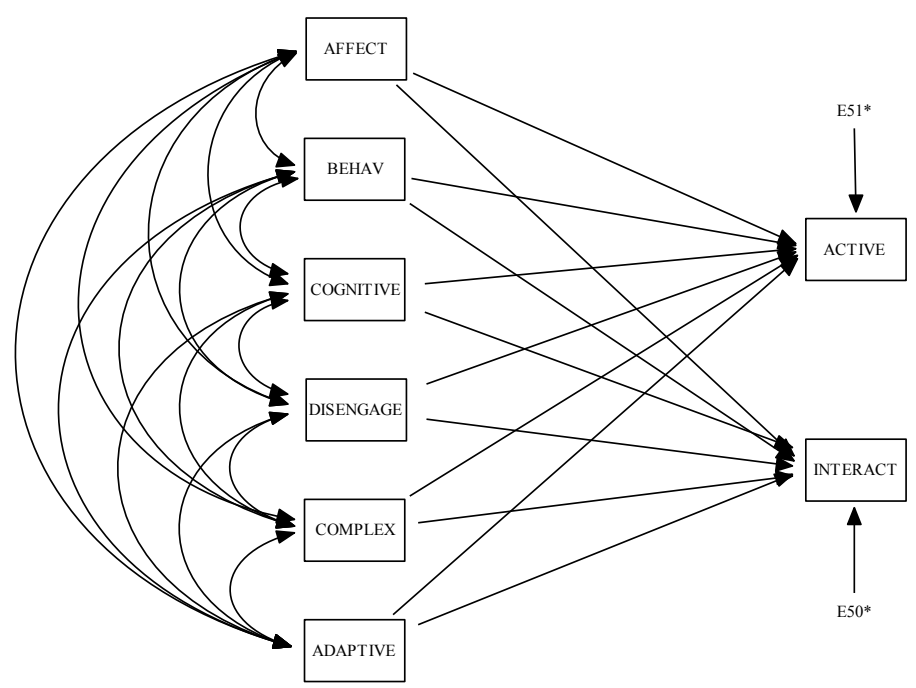

Figure 1. Multivariate model of engagement predicting active and interactive learning.

Note. Curved arrows $=$ correlation. Straight arrows $=$ standardized prediction estimate (i.e. every one unit increase in IV equals an increase in the IV equal to the weight of the parameter).

\section{Results}

Descriptive Statistics. Descriptive statistics were calculated for all study variables. Assumptions checking provided evidence for the relative univariate normality of the instructional strategy and engagement items, with minor positive skew for the least utilized instructional strategies and minor negative skew for the most utilized instructional strategies. Lecture was the most reported instructional strategy. Service learning projects were the least reported instructional strategy. Evidence of univariate normality was treated as evidence for multivariate normality. All study variables were converted to z-scores for subsequent analyses to improve interpretation.

Table 2.

Descriptive statistics for all study variables.

\begin{tabular}{lcccccc}
\hline & Min & Max & M & SD & Skew & Kurt \\
\hline \multicolumn{7}{c}{ Instructional Strategy Items } \\
\hline Small Group Work & 1.00 & 7.00 & 3.68 & 2.12 & 0.06 & -1.32 \\
Artifact Dissection & 1.00 & 7.00 & 2.26 & 1.75 & 1.06 & -0.17 \\
Service Projects & 1.00 & 7.00 & 2.06 & 1.70 & 1.41 & 0.72 \\
Design Projects & 1.00 & 7.00 & 2.16 & 1.78 & 1.26 & 0.19 \\
Build Projects & 1.00 & 7.00 & 2.15 & 1.82 & 1.38 & 0.58 \\
Interdis Design & 1.00 & 7.00 & 2.23 & 1.84 & 1.28 & 0.30 \\
Lecture & 1.00 & 7.00 & 6.14 & 1.47 & -1.91 & 3.07 \\
Guided Practice & 1.00 & 7.00 & 5.04 & 1.81 & -0.70 & -0.42 \\
\hline & 1.00 & 7.00 & 4.50 & 1.39 & -0.25 & -0.47 \\
\hline Affect Engage & 1.00 & 7.00 & 4.05 & 1.20 & -0.07 & 0.02 \\
Behavioral Engage & 2.50 & 7.00 & 5.21 & 0.89 & -0.16 & -0.31 \\
Cognitive Engage & \multicolumn{7}{c}{ Engagement Constructs }
\end{tabular}




\begin{tabular}{lcccccc} 
Disengagement & 1.00 & 7.00 & 3.23 & 1.26 & 0.20 & -0.31 \\
Complex Engage & 1.00 & 7.00 & 4.54 & 1.46 & -0.86 & 0.64 \\
Adaptive Engage & 1.00 & 7.00 & 4.29 & 1.44 & -0.78 & 0.40 \\
\hline
\end{tabular}

Note. $n=346$; Results for the engagement constructs are aggregate scores for the items that align with the specific construct. Results for the instructional strategies scores for the individual items.

Exploratory Factor Analysis

The hypothesized factor structure consisted of eight items representing two concepts: active and interactive learning. The scree plot indicated that the a priori two factor hypothesis was correct. Based on the plot, two factors were rotated using a direct oblimin rotation procedure with Kaiser normalization. The rotated solution, presented in Table 3 along with item anchor meanings, yielded two interpretable factors: $\mathrm{f} 1=$ interactive learning; $\mathrm{f} 2=$ active learning. The interactive learning factor (Eigenvalue $=4.25$ ) accounted for $53.16 \%$ of the item variance and the active learning factor (Eigenvalue $=1.10$ ) accounted for $13.78 \%$ of the total variance. Based on the results, factor scores were saved as variables to be used in subsequent analyses.

Table 3

Factor loadings for rotated two factor solution

\begin{tabular}{lcc}
\hline \multirow{2}{*}{ Instruction } & \multicolumn{2}{c}{ Factor } \\
\cline { 2 - 3 } & 1 & 2 \\
\hline Small Group Work & 0.92 & \\
Artifact Dissection & 0.91 & \\
Service Projects & 0.81 & \\
Design Projects & 0.80 & \\
Build Projects & 0.71 & \\
Interdis Design & 0.48 & \\
Lecture & -0.45 & 0.66 \\
Guided Practice & & 0.30 \\
\hline Note. N = 346; Factor 1 = Interactive Learning; Factor 2 \\
= Active Learning
\end{tabular}

= Active Learning

\section{Bivariate Correlations}

Correlation coefficients were computed among the six engagement variables and the active and interactive learning variables. In line with convention, probability values of less than .05 and .01 are reported (see Table 4). Eighteen of the correlations were statistically significant, with values greater than or less than $+/-.1$ significant at the .05 level and values greater than or less than $+/$ .18 significant at the .01 level. Interactive learning was significantly and positively related to affective engagement, behavioral engagement, disengagement, complex engagement, and adaptive engagement. Active learning was significantly and negatively related to behavioral engagement, complex engagement, and adaptive engagement, and significantly and positively related to cognitive engagement.

Table 4.

Bivariate correlations among all study variables.

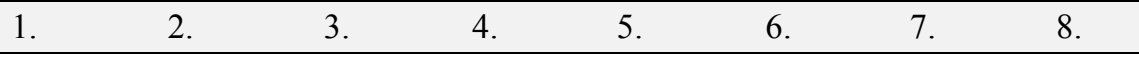




\begin{tabular}{lcccccccc} 
1. Interactive Learning & 1 & & & & & & & \\
2. Active Learning & $-.314^{* *}$ & 1 & & & & & & \\
3. Affect Engage & $.185^{* *}$ & -.055 & 1 & & & & & \\
4. Behavioral Engage & $.365^{* *}$ & $-.122^{*}$ & $.603^{* *}$ & 1 & & & & \\
5. Cognitive Engage & -.063 & $.186^{* *}$ & $.494^{* *}$ & $.487^{* *}$ & 1 & & & \\
6. Disengagement & $.276^{* *}$ & -.082 & -.083 & -.060 & $-.261^{* *}$ & 1 & & \\
7. Complex Engage & $.290^{* *}$ & $-.120^{*}$ & $.205^{* *}$ & $.353^{* *}$ & $.124^{*}$ & .009 & 1 \\
8. Adaptive Engage & $.379^{* *}$ & $-.116^{*}$ & $.252^{* *}$ & $.408^{* *}$ & $.149^{* *}$ & .050 & $.897^{* *}$ & 1 \\
\hline Note. $\mathrm{n}=346 .^{* *}=p<.01 *^{*}=p<.05$ & & & & & & &
\end{tabular}

\section{Structural Equation Modeling}

A structural equation path analytic model (i.e. Byrne, 2006), including six independent variables (engagement constructs) and two dependent variables (active and interactive learning) was examined using EQS 6.2. Results produced acceptable fit statistics, $\chi^{2}(28)=1095.95, p<.05$, $\mathrm{CFI}=.98, \mathrm{RMR}=.03$; SRMR $=03$. See table 5 for a summary of the parameter estimates. Seven of the parameter estimates predicting active and interactive learning from engagement were significant. Controlling for the shared variance among the engagement constructs, behavioral engagement, disengagement, complex engagement, and adaptive engagement were significant and positive predictors of interactive learning $\left(\mathrm{r}^{2}=.32\right)$. Cognitive engagement was a significant negative predictor of interactive learning. Controlling for the shared variance among the engagement constructs, behavioral engagement was a significant negative predictor of active learning and cognitive engagement was a significant positive predictor of active learning $\left(\mathrm{r}^{2}=\right.$ $.10)$.

Table 5 .

Summary of parameter estimates from multivariate structural equation model.

\begin{tabular}{|c|c|c|c|c|c|c|c|c|c|}
\hline & \multirow{2}{*}{ Engagement } & \multicolumn{6}{|c|}{ Correlations } & \multicolumn{2}{|c|}{ Estimates } \\
\hline & & 1. & 2. & 3. & 4. & 5. & 6. & Interactive & Active \\
\hline 1. & Affect Engage & 1 & & & & & & .03 & -.08 \\
\hline 2. & Behavioral Engage & $.60^{*}$ & 1 & & & & & $.37 *$ & $-.21 *$ \\
\hline 3. & Cognitive Engage & $.50 *$ & $.49 *$ & 1 & & & & $-.24 *$ & $.33^{*}$ \\
\hline 4. & Disengagement & -.08 & -.06 & $-.26 *$ & 1 & & & $.22 *$ & -.02 \\
\hline 5. & Complex Engage & $.21 *$ & $.35^{*}$ & $.13 *$ & .01 & 1 & & $.20 *$ & -.09 \\
\hline 6. & Adaptive Engage & $.25 *$ & $.41 *$ & $.15^{*}$ & .05 & $.89 *$ & 1 & $.41 *$ & .03 \\
\hline
\end{tabular}

Note. $\mathrm{N}=346$; Correlations correspond with curved arrows in Figure 1; Prediction estimates correspond with straight arrows in Figure 1. Interactive learning $r^{2}=.32$. Active learning $r^{2}=.10$.

\section{Discussion}

\section{Innovative Instruction in the Engineering Classroom}

Analysis of the descriptive statistics suggested that student self-reported mean levels of instructional strategies roughly aligned with the Borrego et al. (2010) surveys of engineering department chairs. Students reported receiving most of their instruction in the form of lecture and guided practice, with the most common interactive instructional strategy being group work activities in class. However, less frequently students reported other innovative forms of instructional strategies, such as artifact dissection, service projects, design projects, build 
projects, and interdisciplinary design projects. Taking the descriptive statistics and correlation evidence together, results provide evidence that students in the sample were introduced to innovative forms of instructional strategies in their energy-related engineering courses, and that these innovative forms of instructional strategies promoted interactive learning and higher levels of engagement than traditional forms of instruction.

\section{Active and Interactive Learning in the Engineering Classroom}

The results of the factor analysis suggested that the collection of instructional strategies reflect two underlying dimensions of learning, active and interactive. Interpretation of the latent factors produced by the exploratory factory analysis suggest that the instructional strategy items mapped onto Chi's (2009) definitions of active and interactive learning. According to Chi's work, active learning is the process of attending to and selecting relevant information, which maps onto the traditional strategy items (i.e. lecture and guided practice). Interactive learning, on the other hand, is the co-construction of knowledge through collaborative work and problem solving, which maps onto the innovative strategy items (i.e. group work, build projects, etc.). Researchers in engineering education may be able to produce more nuanced and accurate findings by differentiating between active and interactive forms of instruction in their classroom-based surveys, observation protocols, and qualitative coding schemes. For example, Hilpert and Husman (in press), using a combination of observation protocols and classroom running records to study introductory engineering courses, found that engineering educators used active learning strategies to activate prior knowledge for learning and interactive strategies to promote classroom collaboration. The bivariate correlational results of the student surveys reported in the current study support this claim, suggesting that active learning strategies promote cognitive engagement, whereas interactive strategies promote behavioral, complex, and adaptive engagement. These different forms of engagement, and their relationship to different learning processes have important implications for the why and for what purpose of instructional strategy use. These implications are addressed in the following section.

\section{The Multidimensional Relationship between Engagement and Learning}

The results of the multivariate analysis suggest that different combinations of classroom engagement are related to active and interactive learning processes. Active learning seems to be related to a behaviorally inert form of learning that promotes slow and deliberate conscious processing of new information. Interactive learning seems to be related to a behaviorally dynamic form of learning that promotes rapid, automatic processing. Evans (2008) argues for a dual processing model of thinking and reasoning with two overlapping systems. These two forms of thinking align with the profiles produced in the current study. In Evan's (2008) formulation, system one is responsible for rapid sense making and happens unconsciously whereas system two is responsible for systematic processing happens consciously (i.e. Kahneman, 2011). These two systems are not mutually exclusive. Although cognitive scientists are not clear on the mechanism of interaction between them, it is clear that slow conscious thinking can become automatic with practice and experience, and that system two can interfere with system one to prevent errors associated with automatic processing (Evans, 2008).

The engagement profiles extracted from our SEM suggest that during active learning, students may be slowly, consciously processing new information through attending to and selecting new information. During interactive learning, students maybe rapidly, unconsciously processing information though naturalistic interaction and exploration. During active learning, students may 
be operating individually, asking themselves questions and maintaining attentional control. During interactive learning, they may be operating as a part of a larger classroom system, cognitively disengaged from conscious attentional control, but intuitively applying their knowledge to explore possible solutions to problems and co-construct knowledge. Both are integral to success in the work place. For example, fast, unconscious processing appears to be what firefighters, military commanders, intelligence agents, and engineers use to solve problems in the field (Kline, 1999). Slow, unconscious processing, other the other hand, appears to be what is related to conceptual change, or the revision of mental models and frames of reference for examining problems (i.e. Ohlsson, 2009).

The current results suggest that active learning may promote system two thinking, and interactive learning may promote system one thinking, providing empirical evidence that traditional instructional strategies may best for developing and changing mental models, and innovative instructional strategies may be best for teaching the types of naturalistic decision making and critical thinking skills required for authentic work. When viewed within the context of previous research (Hilpert \& Husman, in press), it seems that traditional instructional strategies can help students to deliberately add to or revise mental models by activating prior knowledge, and innovative instructional strategies create interactive classroom systems that can help students learn to intuitively apply new knowledge. These two processes are not orthogonal, and the findings do not suggest that students are only using one or the other during active and interactive learning. However, the engagement profiles produced here suggest that one system or the other may dominate during different types of learning, and, accordingly, that different instructional strategies are more appropriate for different instructional goals. These findings can help engineering educators develop generalizable, rule based conclusions about how to shape learning experiences for students using research based instructional strategies.

\section{Limitations and Future Directions}

The limitations of the current study include typical concerns about the use of survey research in classrooms, including self-selection bias, desirability during self-report, questions about generalizability, and other common limitations that hardly need explication. Perhaps more importantly, the instructional strategy categories used here do not include many types of research based instructional strategies offered in the literature, for example think pair share, just in time teaching and so on (Borrego, et al., 2013). Some of these are probably subsumed within the group work category utilized in the current study, but more of them could and should be studied separately in future survey research to examine if the active and interactive dimensions hold up within more comprehensive frameworks of research based instructional strategies. Research along these lines could also replicate the engagement profiles produced here. The current findings suggest that using linear combinations of engagement variables in statistical analyses may portray a more accurate picture of the shared variation between instruction and engagement. Future research should examine if the effect sizes produced in the current study, which are larger than those produced from previous research (Gasiewski et al., 2012; Hilpert \& Husman, in press; Marchand \& Gutierrez, in press), are replicable across time and context. Finally, these findings, taken within the context of previous research, point to the possibility that different research based instructional strategies may produce different forms of dynamic classroom processes, with multiple target levels of analysis. Future research should continue to examine engineering science classrooms from a complex systems perspective, unpacking the impact of instructional strategies on both individual and interactive levels of analysis to provide a more complete picture 
of how the implementation of research based instructional strategies influences student learning, thinking, and problem solving.

\section{References}

American Society for Engineering Education (ASEE). (2009). Creating a Culture for Scholarly and Systematic Innovation in Engineering Education: Ensuring U.S. engineering has the right people with the right talent for a global society. Washington, DC: American Society for Engineering Education.

Borrego, M., Culter, S., Prince, M., Henderson, C., \& Froyd J. E. (2013). Fidelity of implementation of research-based instructional strategies (RBIS) in engineering science courses. Journal of Engineering Education, 102, 294-425.

Borrego, M., Froyd, J. E., \& Hall, T. S. (2010). Diffusion of engineering education innovations: A survey of awareness and adoption rates in U.S. engineering departments. Journal of Engineering Education. 99(3), 185-207

Bryne, B. M. (2006). Structural equation modeling with EQS: Basic concepts, applications, and programming. Lawrence Erlbaum Associates, Mahwah, NJ.

Caldwell, J. E. (2007). Clickers in the large classroom: Current research and best-practice tips. Life Sciences Education, 6(1), 9-20.

Chen, H. L., Lattuca, L. R., Hamilton, E. R. (2008). Conceptualizing Engagement: Contribution of Faculty to Student Engagement in Engineering. Journal of Engineering Education, 3, 339-353.

Chi, M. T. H. (2009). Active-Constructive-Interactive: A conceptual framework for differentiating learning activities. Topics in Cognitive Science, 1, 73-105.

Corno, L., \& Mandinach, E. B. (2004). What have we learned about engagement in the last twenty years? A Volume in: Research on Sociocultural Influences on Motivation and Learning, (pp. 297-326), Information Age Publishing, Inc.

Evans, St. B. T. J. (2008). Dual processing accounts of reasoning, judgement, and decision making. Annual Review of Psychology, 59, 255-78.

Felder, Richard M., \& Brent, Rebecca. (2009). Active Learning: An Introduction. ASQ Higher Education Brief, 2(4).

Fredricks, J. A., Blumenfeld, P. C., \& Paris, A. H., (2004). School Engagement: Potential of the Concept, State of the Evidence. Review of Educational Research, 74(1), 59-109.

Freeman, S., Eddy, S. L., McDonough, M., Smith, M. K., Okoroafor, N., Jordt, H., \& Wenderoth, M. P. (2014). Active learning increases student performance in science, engineering, and mathematics. PNAS, 111(23), 8410-8415.

Gasiewski, J. A., Eagan, M. K., Garcia, G. A., Hurtado, S., \& Chang, M. J. (2012). From gatekeeping to engagement: A multicontextual, mixed method study of student academic engagement in introductory STEM courses. Research in Higher Education, 53, 229-261.

Green, S. B. \& Salkind, N. J. (2011). Using SPSS for windows and machintosh: Analyzing and understanding data $\left(6^{\text {th }}\right.$ ed). Pearson. 
Hake, R. R. (1998). Interactive-Engagement vs. Traditional Methods: A Six-Thousand-Student Survey of Mechanics Test Data for Introductory Physics Courses. American Journal of Physics, 66(1), 64-74.

Henderson, C., Beach, A., \& Finkelstein, N., (2011). Facilitating change in undergraduate STEM instructional practices: An analytic review of the literature. Journal of Research in Science Teaching, 48(8), 952-984.

Hilpert, J. \& Holiday, R., (2015). Using Havel-Hakimi to graph classroom networks. Proceeding published in the annual proceedings of the American Society of Engineering Educators annual conference, Seattle, WA.

Hilpert, J. \& Husman, J. (2015). A group-level framework for emergent properties of interactive learning. Proceeding published from the invited NSF grantees session of the American Society of Engineering Educators annual conference to be held in Seattle, WA.

Hilpert, J. \& Husman, J. (2016). Student Perceptions of the Emergent Properties of Interactive Learning during Classroom Group Work. Paper accepted as poster to the annual meeting of the American Education Research Association, Washington, DC.

Hilpert, J. \& Husman, J. (in press). Instruction and engagement in the engineering classroom: The complexity underlying small effect sizes. (Empirical article accepted for publication; Educational Psychology).

Kahneman, D. (2011). Thinking, fast and slow. Macmillan.

Klein, G. A. (1999). Sources of power: How people make decisions. MIT press.

Knight, J. K., \& Wood, W. B. (2005). Teaching more by lecturing less. Cell Biology Education, 4(4) 298-310.

Kuh G.D. (2002) The National Survey of Student Engagement: Conceptual Framework and Overview of Psychometric Properties. Center for Postsecondary Research, Indiana University, Bloomington. Available at:

http://nsse.iub.edu/pdf/psychometric framework_2002.pdf

Lawson, M. A., \& Lawson, H. A. (2013). New conceptual frameworks for student engagement research, policy, and practice. Review of Educational Research, 0034654313480891.

Marchand, G. C., \& Gutierrez, A., (in press). Processes involved in perceived instructional support, task values, and engagement in graduate education. The Journal of Experimental Education.

McKeachie, W., \& Svinicki, M. (2014) Teaching tips: Strategies, research, and theory for college and university teachers $\left(14^{\text {th }} \mathrm{ed}\right)$. Wadsworth, Cengage Learning.l

National Research Council. (2012). Discipline-Based Educational Research: Understanding and Improving Learning in Undergraduate Science and Engineering. Washington, DC: National Academies Press.

Ohland, M. W., Sheppard, S. D., Lichtenstein, G., Ozgur, E., Chachra, D., \& Layton, R. A. (2008). Persistence, engagement, \& Migration in Engineering Programs. Journal of Engineering Education, 3, 259-278.

Ohlsson, S. (2009). Resubsumption: A possible mechanism for conceptual change and belief revision. Educational Psychologist, 44(1), 20-40.

Pentland, A., (2014). Social physics: How good ideas spread - the lessons from a new science. The Penguin Press.

Prince, M. (2004). Does active learning work? A review of the research. Journal of Engineering Education, 93(3), 223-231. 
Seymour, E., \& Hewitt, N. M., (1997). Talking about leaving: Why undergraduates leave the sciences. Boulder, CO: Westview Press.

Skinner, E. A., Furrer, C., Marchand, G., \& Kindermann, T. A. (2008). Engagement and disaffection in the classroom: Part of a larger motivational dynamic? Journal of Educational Psychology, 100(4), 765-781.

Tabachnick, B. G., \& Fidell, L. S. (2012). Using Multivariate Statistics ( $6^{\text {th }}$ ed). Pearson.

Wang, Ze, Bergin, C., \& Bergin, D., (2014). Measuring Engagement in $4^{\text {th }}$ to $12^{\text {th }}$ Grade Classrooms: The Classroom Engagement Inventory. School Psychology Quarterly, 29(4), p. 517-35. doi: 10.1037/spq0000050. Epub 2014 Apr 7.

Zeilik, M., \& Morris, V. J. (2004). The impact of cooperative quizzes in a large introductory astronomy course for non-science majors. Astronomy Education Review, 3(1), 51-61. 